\title{
Cosmic microwave background statistics for a direction-dependent primordial power spectrum
}

\author{
Anthony R. Pullen and Marc Kamionkowski \\ California Institute of Technology, Mail Code 130-33, Pasadena, California 91125, USA
}

(Received 7 September 2007; published 21 November 2007)

\begin{abstract}
Statistical isotropy of primordial perturbations is a common assumption in cosmology, but it is an assumption that should be tested. To this end, we develop cosmic microwave background statistics for a primordial power spectrum that depends on the direction, as well as the magnitude, of the Fourier wave vector. We first consider a simple estimator that searches in a model-independent way for anisotropy in the square of the temperature (and/or polarization) fluctuation. We then construct the minimum-variance estimators for the coefficients of a spherical-harmonic expansion of the directional dependence of the primordial power spectrum. To illustrate, we apply these statistics to an inflation model with a quadrupole dependence of the primordial power spectrum on direction and find that a power quadrupole as small as $2.0 \%$ can be detected with the Planck satellite.
\end{abstract}

DOI: 10.1103/PhysRevD.76.103529

PACS numbers: $98.80 .-\mathrm{k}$

\section{INTRODUCTION}

It is well known that the homogeneity and isotropy of the Universe are only approximate. There are departures from homogeneity and isotropy that are now well quantified by measurements of the cosmic microwave background (CMB) and galaxy surveys. In current cosmological theory, the notions of homogeneity and isotropy have been superseded by the notions of statistical homogeneity and isotropy. The density of matter may differ from one point in the Universe to another, but the distribution of matter is described as a realization of a random field with a variance that is everywhere the same and the same in every direction. This is generally the prediction of structure-formation models, and, in particular, of inflationary models.

Still, statistical isotropy and homogeneity are assumptions that can be tested quantitatively, and the precision with which they can be tested is improving rapidly with the still-accumulating wealth of cosmological data. Preliminary (and controversial) indications for a preferred direction in the CMB [1-4] have recently motivated the study of departures from statistical isotropy. Subsequent theoretical work has shown that although statistical isotropy is a generic prediction of inflation, inflation models can in fact be constructed to violate statistical isotropy [514]. Dark-energy models might also accommodate departures from statistical isotropy $[15,16]$. These models provide useful straw men against which the success of the standard inflationary predictions of statistical isotropy can be quantified.

The growing interest in such models motivates us to study generalized tests for statistical isotropy. In a statistically isotropic Universe, the primordial distribution of matter is a realization of a random field in which Fourier modes of the density field have variances, a power spectrum $P(\mathbf{k})$, that depend only on the magnitude $k$ of the wave vector $\mathbf{k}$. If we drop the assumption of statistical isotropy, the power spectrum will depend on the direction $\hat{\mathbf{k}}$ as well. If $\delta(\mathbf{k})$ is the Fourier amplitude of the fractional density perturbation, then the power spectrum is defined by

$$
\left\langle\delta(\mathbf{k}) \delta^{*}\left(\mathbf{k}^{\prime}\right)\right\rangle=\delta_{D}\left(\mathbf{k}-\mathbf{k}^{\prime}\right) P(\mathbf{k}),
$$

where the angle brackets denote an average over all realizations of the random field, and $\delta_{D}$ is a Dirac delta function; note that we are still preserving the assumption that different Fourier modes are uncorrelated. The most general power spectrum can then be written,

$$
P(\mathbf{k})=A(k)\left[1+\sum_{L M} g_{L M}(k) Y_{L M}(\hat{\mathbf{k}})\right]
$$

where $Y_{L M}(\hat{\mathbf{k}})$ (with $L \geq 2$ ) are spherical harmonics, and $g_{L M}(k)$ quantify the departure from statistical isotropy as a function of wave number $k$. Since the density field is real, Fourier modes for $\mathbf{k}$ are related to those of $-\mathbf{k}$, in such a way that the multipole moment $L$ must be even. In the limit $g_{L M}(k) \rightarrow 0$, we recover the usual statistically isotropic theory with power spectrum $A(k)$. The implementation, Eq. (2), of power anisotropy is motivated in part by the inflationary model of Ref. [10], which predicts $g_{2 M}(k) \neq 0$.

Here we consider several CMB tests for statistical isotropy. The first, which we refer to as "power multipole moments," is a simple and intuitive estimator that involves measurement of the multipole moments of the square of the temperature/polarization fields. ${ }^{1}$ As an example, we apply this statistic to an inflationary model [10] that predicts a quadrupole in the matter power spectrum.

\footnotetext{
${ }^{1}$ There has already been some evidence for a dipole in the $\mathrm{CMB}$ power $[2,4]$ that is analogous to the higher multipole moments that we are considering here, but which cannot be due to anisotropy in the primordial power spectrum because it has $L=1$. There have also been searches [3] for anisotropy along the lines considered here, and Refs. [17,18] discuss similar statistics.
} 
Although power multipole moments provide a nice model-independent test for departures from statistical isotropy, more sensitive probes can be developed if the particular form of the departure is specified. To illustrate, we thus construct the minimum-variance estimators for the anisotropy coefficients $g_{L M}(k)$ under the assumption that they are constants. The naive power multipole moments, although intuitively simple, co-add a number of modes with equal weight. The minimum-variance estimator coadds modes with weights that depend on their signal to noise, so that (as the name suggests) the variance of the estimator is minimized. We show that this statistic provides a far stronger probe for the $g_{L M}$ 's.

The plan of our paper is as follows: Sec. II reviews some CMB basics. Section III calculates the correlations of CMB spherical-harmonic coefficients if there are departures from statistical isotropy. As we discuss there, the power spectrum $C_{l}$, which describes the two-point CMB statistics if there is statistical isotropy, is generalized to a set of moments $D_{l l^{\prime}}^{L M}$ if statistical isotropy is broken. In Sec. IV, we introduce and calculate the power multipole moments and calculate the standard errors with which these moments can be recovered. We apply this statistic to a quadrupole in the matter power spectrum, calculating the sensitivities of several CMB experiments to such a quadrupole. Section V discusses minimum-variance estimators for the quantities $D_{l l^{\prime}}^{L M}$ that parametrize the departures $g_{L M}(k)$ from statistical isotropy. We then construct from these the minimum-variance estimators for the quadrupole moments of the primordial power spectrum, calculate their variance, and evaluate their sensitivity to departures from statistical isotropy. We make some concluding remarks in Sec. VI. Throughout the main body of the paper, we discuss statistics for only a temperature map, in order to make the presentation clear. The Appendix generalizes to include the full temperature-polarization information. Our numerical results are for a full temperature-polarization map, as well as for temperature or polarization alone.

\section{PRELIMINARIES}

A CMB experiment provides the temperature $T(\hat{\mathbf{n}})$ as a function of position $\hat{\mathbf{n}}$ on the sky. The map $T(\hat{\mathbf{n}})$ can be expanded in terms of spherical harmonics $Y_{l m}(\hat{\mathbf{n}})$,

$$
a_{l m}=\frac{1}{T_{0}} \int d \hat{\mathbf{n}} Y_{l m}^{*}(\hat{\mathbf{n}}) T(\hat{\mathbf{n}}) .
$$

The $a_{l m}$ 's are Gaussian random variables, and if there is statistical isotropy, then they are statistically independent for different $l$ and $m:\left\langle a_{l m} a_{l^{\prime} m^{\prime}}^{*}\right\rangle=C_{l} \delta_{l l^{\prime}} \delta_{m m^{\prime}}{ }^{2}$. The set of $C_{l}$ 's is the CMB temperature power spectrum. We will see

\footnotetext{
${ }^{2}$ Strictly speaking, it is not the $a_{l m}$ 's that are statistically independent, but rather their real and imaginary parts.
}

that when statistical isotropy is violated, there are correlations induced between $a_{l m}$ 's for different $l$ and $m$ [10]. If there is statistical isotropy, the two-point autocorrelation function is

$$
C\left(\hat{\mathbf{n}}, \hat{\mathbf{n}}^{\prime}\right)=\left\langle T(\hat{\mathbf{n}}) T\left(\hat{\mathbf{n}}^{\prime}\right)\right\rangle=T_{0}^{2} \sum_{l} \frac{2 l+1}{4 \pi} C_{l} P_{l}\left(\hat{\mathbf{n}} \cdot \hat{\mathbf{n}}^{\prime}\right)
$$

i.e., the correlation function depends only on the separation between the two points. If statistical isotropy is violated, this is not necessarily true.

\section{OFF-DIAGONAL CORRELATIONS FOR ANISOTROPIC POWER}

Consider a primordial matter power spectrum $P(\mathbf{k})$ given by Eq. (2). We expand $T(\hat{\mathbf{n}})$ in $\mathbf{k}$ space in the form

$$
\frac{T}{T_{0}}(\hat{\mathbf{n}})=\int d^{3} k \sum_{l}(-i)^{l}(2 l+1) P_{l}(\hat{\mathbf{k}} \cdot \hat{\mathbf{n}}) \delta(\mathbf{k}) \Theta_{l}(k)
$$

where $\Theta_{l}(k)$ is the contribution to the $l$ th temperature moment from wave vector $\mathbf{k}$. With these conventions, $\Theta_{l}(k)$ is real. With our expression, Eq. (2), we can write the covariance matrix as

$$
\left\langle a_{l m} a_{l^{\prime} m^{\prime}}^{*}\right\rangle=\delta_{l l^{\prime}} \delta_{m m^{\prime}} C_{l}+\sum_{L M} \xi_{l m l^{\prime} m^{\prime}}^{L M} D_{l l^{\prime}}^{L M}
$$

Here, the set of $C_{l}$ 's, given by

$$
C_{l}=(4 \pi)^{2} \int_{0}^{\infty} d k k^{2} A(k)\left[\Theta_{l}(k)\right]^{2},
$$

is the usual CMB power spectrum for the case of statistical isotropy. Departures from statistical isotropy introduce the second term, where

$$
D_{l l^{\prime}}^{L M}=(4 \pi)^{2}(-i)^{l-l^{\prime}} \int_{0}^{\infty} d k k^{2} A(k) g_{L M}(k) \Theta_{l}(k) \Theta_{l^{\prime}}(k)
$$

and

$$
\begin{aligned}
\xi_{l m l^{\prime} m^{\prime}}^{L M} & =\int d \hat{\mathbf{k}} Y_{l m}^{*}(\hat{\mathbf{k}}) Y_{l^{\prime} m^{\prime}}(\hat{\mathbf{k}}) Y_{L M}(\hat{\mathbf{k}}) \\
& =(-1)^{m^{\prime}}\left(G_{l l^{\prime}}^{L}\right)^{1 / 2} C_{l m l^{\prime},-m^{\prime}}^{L M}
\end{aligned}
$$

where $C_{l m l^{\prime} m^{\prime}}^{L M}$ are Clebsch-Gordan coefficients, and

$$
G_{l l^{\prime}}^{L} \equiv \frac{(2 l+1)\left(2 l^{\prime}+1\right)}{4 \pi(2 L+1)}\left(C_{l 0 l^{\prime} 0}^{L 0}\right)^{2} .
$$

Throughout, we use uppercase indices $L M$ for power anisotropies, and lowercase indices $\mathrm{lm}$ for temperature/ polarization anisotropies. For $L$ even, $\xi_{l m l^{\prime} m^{\prime}}$ are nonvanishing only for $l-l^{\prime}$ even, and so the $D_{l l^{\prime}}^{L M}$ are real. 
Equations (8) and (9) agree with similar results in Ref. [18], and they recover the results of Ref. [10] for $L=2$.

If primordial perturbations are statistically isotropic and Gaussian, then the statistics of the CMB temperature map are specified fully by the power spectrum, the set of $C_{l}$ 's. If primordial perturbations have a departure from statistical isotropy that can be written in terms of spherical harmonics $Y_{L M}(\hat{\mathbf{k}})$, then the two-point statistics are described additionally by the set of multipole moments $D_{l l^{\prime}}^{L M}$. These quantities are thus the generalization of the $C_{l}$ 's if there is statistical anisotropy.

\section{POWER MULTIPOLE MOMENTS}

\section{A. Theoretical predictions}

It is natural to expect that a spherical-harmonic pattern of anisotropy in the matter power spectrum manifests itself in a similar pattern in the CMB power. It is thus natural to consider a set of "power multipole moments,"

$$
b_{L M}=\frac{1}{T_{0}^{2}} \int d \hat{\mathbf{n}} Y_{L M}^{*}(\hat{\mathbf{n}})\left\langle T^{2}\right\rangle(\hat{\mathbf{n}}),
$$

where $\left\langle T^{2}\right\rangle(\hat{\mathbf{n}})=C(\hat{\mathbf{n}}, \hat{\mathbf{n}})$ is the expectation value of the square of the temperature at position $\hat{\mathbf{n}}$ in the sky; it is the autocorrelation function at zero lag. With this statistic, we simply look for anisotropies in the power. These statistics have several advantages. In addition to having a form familiar from similar statistics [e.g., Eq. (3)] for temperature fluctuations, they have simple analytic expressions in terms of $P(\mathbf{k})$. There are also (as we show below), relatively simple expressions for the cosmic-variance-induced and instrumental-noise-induced errors in the measurement of these statistics.

The variance $\left\langle T^{2}\right\rangle(\hat{\mathbf{n}})$ as a function of position $\hat{\mathbf{n}}$ is given by

$$
\frac{\left\langle T^{2}\right\rangle(\hat{\mathbf{n}})}{T_{0}^{2}}=\sum_{l m l^{\prime} m^{\prime}}\left\langle a_{l m} a_{l^{\prime} m^{\prime}}^{*}\right\rangle Y_{l m}(\hat{\mathbf{n}}) Y_{l^{\prime} m^{\prime}}^{*}(\hat{\mathbf{n}})
$$

We put this into Eq. (11) and use

$$
\sum_{m m^{\prime}} C_{l m l,-m^{\prime}}^{L M} C_{l m l^{\prime},-m^{\prime}}^{L^{\prime} M^{\prime}}=\delta_{L L^{\prime}} \delta_{M M^{\prime}},
$$

to obtain (for $L \geq 2$ )

$$
b_{L M}=\sum_{l l^{\prime}} G_{l l^{\prime}}^{L} D_{l l^{\prime}}^{L M}
$$

\section{B. Statistical noise}

We now calculate the standard error, due to cosmic variance and instrumental noise, with which the power multipole moments can be measured. To do so, we con- sider a full-sky map $T^{\text {map }}(\hat{\mathbf{n}})$ of the temperature in $N_{\text {pix }}$ equal-area pixels. The temperature in each pixel receives contributions from signal and from noise. Thus, in pixel $i$, $T^{\text {map }}=T\left(\hat{\mathbf{n}}_{i}\right)+T_{i}^{\mathrm{n}}$, where $T\left(\hat{\mathbf{n}}_{i}\right)$ is the temperature measured in pixel $i$, which will be the signal temperature smoothed by a Gaussian beam of full width half maximum (FWHM) $\theta_{\mathrm{FWHM}}$, plus a noise $T_{i}^{\mathrm{n}}$. We assume that the noise is isotropic and that the noises in different pixels are uncorrelated with variance $\sigma_{T}^{2}$ : i.e., $\left\langle T_{i}^{\mathrm{n}} T_{j}^{\mathrm{n}}\right\rangle=\sigma_{T}^{2} \delta_{i j}$. The power spectrum for the map is thus $C_{l}^{\text {map }}=\left|W_{l}\right|^{2} C_{l}+C_{l}^{\mathrm{n}}$, where $C_{l}^{\mathrm{n}}=\left(4 \pi / N_{\text {pix }}\right) \sigma_{T}^{2}$ is the noise power spectrum, and $W_{l}$ is a window function that takes into account the effects of beam smearing; for a Gaussian beam of FWHM $\theta_{\mathrm{FWHM}}$, it is $W_{l}=\exp \left(-l^{2} \sigma_{b}^{2} / 2\right)$ with $\sigma_{b}=\theta_{\mathrm{FWHM}} / \sqrt{8 \ln 2}=$ $0.00742\left(\theta_{\mathrm{FWHM}} / 1^{\circ}\right)$.

Since the instrumental noise is isotropic by assumption, we get an unbiased estimator for $b_{L M}$ (for $L \geq 2$ ) from

$$
\hat{b}_{L M}^{\mathrm{map}}=\frac{1}{T_{0}^{2}} \int d \hat{\mathbf{n}} Y_{L M}^{*}(\hat{\mathbf{n}})\left[T^{\mathrm{map}}(\hat{\mathbf{n}})\right]^{2} .
$$

Cosmic variance and instrumental noise induce a variance in the $b_{L M}$ 's, which we define as

$$
\Xi^{L M} \equiv\left\langle\hat{b}_{L M} \hat{b}_{L M}\right\rangle
$$

where we have assumed the null hypothesis, $g_{L M}=0$. For this null hypothesis of a statistically isotropic Gaussian random map,

$$
\begin{aligned}
& \Xi^{L M}=\frac{2}{T_{0}^{4}} \int d \hat{\mathbf{n}} d \hat{\mathbf{n}}^{\prime} C^{\operatorname{map}}\left(\hat{\mathbf{n}}, \hat{\mathbf{n}}^{\prime}\right) C^{\operatorname{map}}\left(\hat{\mathbf{n}}, \hat{\mathbf{n}}^{\prime}\right) Y_{L M}(\hat{\mathbf{n}}) Y_{L M}^{*}\left(\hat{\mathbf{n}}^{\prime}\right) \\
& =2 \sum_{l l^{\prime}} G_{l l^{\prime}}^{L} C_{l}^{\mathrm{map}} C_{l^{\prime}}^{\mathrm{map}} \text {, }
\end{aligned}
$$

where $C^{\text {map }}\left(\hat{\mathbf{n}}_{1}, \hat{\mathbf{n}}_{2}\right)$ is the two-point correlation function for the map, obtained from the expression, Eq. (4), for the correlation function by replacing $C_{l}$ by $C_{l}^{\text {map }}$, and we have used $\sum_{m m^{\prime}}\left(C_{l m l^{\prime} m^{\prime}}^{L M}\right)^{2}=1$. Note that the absence of any $M$ dependence of $\Xi_{\mathrm{AA}^{\prime}}^{L M}$ is as we expected. Moreover, it follows from Eq. (13) that the estimators for the different $b_{l m}$ 's are uncorrelated: $\left\langle\hat{b}_{L M} \hat{b}_{L^{\prime} M^{\prime}}\right\rangle \propto \delta_{L L^{\prime}} \delta_{M M^{\prime}}$.

Given a power spectrum of the form Eq. (2), specified by the functions $g_{L M}(k)$, predictions for the $b_{L M}^{\text {map }}$ can be evaluated with Eq. (14) replacing $D_{l l^{\prime}}^{L M}$ in that equation by $D_{l l^{\prime}}^{L M \text {,map }}=D_{l l^{\prime}}^{L M} W_{l} W_{l^{\prime}}$ and evaluating the $D_{l l^{\prime}}^{L M}$ with Eq. (8). The $b_{L M}^{\mathrm{map}}$ can then be measured using Eq. (15) with variances given by Eq. (17).

\section{A worked example}

As a simple example, suppose the $g_{L M}(k)$ are constants, independent of $k$. We can then take $g_{L M}$ outside the integral in Eq. (8). An estimator for $g_{L M}$ is then $\hat{g}_{L M}=$ $\hat{b}_{l m}^{\text {map }} /\left(b_{l m}^{\text {map }} / g_{L M}\right)$. Defining $F_{l l^{\prime}} \equiv D_{l l^{\prime}}^{L M} / g_{L M}$ for this case, the variance with which each $g_{L M}$ can be measured 
TABLE I. The standard error $\sigma_{g_{2 M}}$ to the amplitude of a quadrupole anisotropy in the matter power spectrum for different experiments. The instrumental temperature and polarization noises and beam width are listed for each experiment. We show results for the power multipole moments (pmm) for TT only, EE only, and the full result. We also show in the last three columns $\sigma_{g_{2 M}}^{\mathrm{mv}}$ from the minimum-variance estimator for each experiment, for TT only, EE only, and the full result.

\begin{tabular}{lccccccccc}
\hline \hline Experiment & $\sigma_{T}(\mu \mathrm{K})$ & $\sigma_{P}(\mu \mathrm{K})$ & $\theta_{\mathrm{FWHM}}$ & $\sigma_{g_{2 M}}^{\mathrm{pmm}}(\mathrm{TT})$ & $\sigma_{g_{2 M}}^{\mathrm{pmm}}(\mathrm{EE})$ & $\sigma_{g_{2 M}}^{\mathrm{pmm}}$ (total) & $\sigma_{g_{2 M}}^{\mathrm{mv}}(\mathrm{TT})$ & $\sigma_{g_{2 M}}^{\mathrm{mv}}(\mathrm{EE})$ & $\sigma_{g_{2 M}}^{\mathrm{mv}}($ total $)$ \\
\hline WMAP & 30.0 & 42.6 & $21^{\prime}$ & 1.3 & 11 & 1.2 & 0.024 & 2.4 & 0.024 \\
Planck & 13.1 & 26.8 & $5^{\prime}$ & 1.6 & 0.16 & 0.16 & 0.0052 & 0.033 & 0.0050 \\
EPIC & 0.021 & 0.068 & $52^{\prime}$ & 1.2 & 0.55 & 0.42 & 0.016 & 0.019 & 0.011 \\
Cosmic variance & 0 & 0 & 0 & 1.8 & 0.014 & 0.014 & & & \\
\hline \hline
\end{tabular}

is then

$$
\sigma_{g_{L M}}^{2}=\frac{2 \sum_{l l^{\prime}} G_{l l^{\prime}}^{L} C_{l}^{\text {map }} C_{l^{\prime}}^{\text {map }}}{\left[\sum_{l l^{\prime}} G_{l l^{\prime}}^{L} F_{l l^{\prime}} W_{l} W_{l^{\prime}}\right]^{2}}
$$

Moreover, the measured $g_{L M}$ are statistically independent as a consequence of the statistical independence of the $\hat{b}_{L M}$.

To illustrate, we apply this result to an inflationary model [10] that has a power spectrum with a quadrupole dependence on the angle. ${ }^{3}$ We use the $\Theta_{l}(k)$ calculated by CMBFAST [19] to obtain $F_{l l^{\prime}}$. We assume only scalar perturbations and the current best-fit cosmological parameters.

The numerical results are given in Table I, but before reviewing them, we provide some very rough estimates to get some feel for the numbers. To do so, ignore instrumental noise and suppose that $W_{l}=1$ for all $l \leq l_{\max }$. For $L=$ $2, F_{l l^{\prime}} \neq 0$ only for $l^{\prime}=l$ or $l^{\prime}=l \pm 2$. Moreover, for these combinations of $l l^{\prime}$ and for $l \gg 2$, we approximate the numerical results (which we use for the numerical results in Table I) for $F_{l l^{\prime}}$ as $F_{l, l+2} \simeq-0.5 C_{l}$. Also, $\left(C_{l 0 l 0}^{20}\right)^{2} \sim(5 / 8) l^{-1}$ for $l \gg 1$, and $\left(C_{l 0(l \pm 2) 0}^{20}\right)^{2}$ is 1.5 times as large. Equation (18) can then be approximated $\sigma_{g_{2 M}}^{2} \sim$ $256 \pi\left[\sum_{l} l\left(C_{l}\right)^{2}\right] /\left[\sum_{l} l C_{l}\right]^{2}$. If the power spectrum has the form $C_{l} \propto l^{-2}$ (a very rough approximation to the temperature power spectrum for $l \lesssim 1000)$, then $\sigma_{g_{2 M}}^{2} \sim$ $128 \pi l_{\min }^{-2}\left[\ln \left(l_{\max } / l_{\min }\right)\right]^{-2}$. For example, using $l_{\min }=2$ and $l_{\max }=1000$ yields $\sigma_{g_{2 M}} \sim 1.23$.

Of course, there is nothing about the derivation of Eq. (18) that is specific to a temperature map, and this result can be applied equally well, e.g., to the E-mode polarization. If we approximate the polarization power spectrum by $C_{l} \sim$ const, then we find $\sigma_{g_{2 M}}^{2} \simeq 512 \pi l_{\max }^{-2}$, or $\sigma_{g_{2 M}} \sim 5 \times 10^{-2}$ for $l_{\max } \simeq 1000$.

We now return to the numerical results for $\sigma_{g_{2 M}}$ listed in Table I for the Wilkinson Anisotropy Probe (WMAP) [20], which has now collected three years of data, the Planck satellite [21], to be launched in 2008, and EPIC [22], a

\footnotetext{
${ }^{3}$ Note that our $g_{20}$ is $(2 / 3) \sqrt{4 \pi / 5} g_{*}$, where $g_{*}$ is the coefficient in Ref. [10] of $(\hat{\mathbf{k}} \cdot \hat{\mathbf{z}})^{2}$ if the preferred direction is taken to be $\hat{\mathbf{z}}$.
}

satellite mission currently under study. The parameters assumed for each model are listed, as well as results obtained using Eq. (14) assuming only TT is used or EE only. The Appendix generalizes Eq. (14) to the case where the full temperature polarization is used (including the TE correlation), and we present numerical results for this case in the Table as well. We also list results, labeled "CVO" (cosmic variance only), for a hypothetical experiment that has perfect angular resolution and no instrumental noise. These numbers are for hypothetical full-sky experiments, but a realistic experiment will likely only be able to use $\sim 65 \%$ of the sky for cosmology. If so, then each estimate for $\sigma_{g_{2 M}}$ must be increased by a factor $(0.65)^{-1 / 2}$, about $25 \%$. We also note that the theory cannot specify the direction $\hat{\mathbf{e}}$ of the quadrupole, and so a search for a quadrupole would require evaluation of all five $g_{2 M}$ 's. A " $3 \sigma$ " detection would thus require that the sum of the squares of the $g_{2 M}$ 's needs to exceed $\left(3 \sigma_{g_{2 M}}\right)^{2}$, which is independent of $M$.

The order of magnitude that we would expect for $\sigma_{g_{2 M}}$ is $\sim N_{\text {pix }}^{-1 / 2}$, where $N_{\text {pix }} \sim l_{\max }^{2}$ is the number of resolution elements on the sky, comparable to the precision with which one can measure the variance (the monopole) of the temperature-fluctuation amplitude. The numerical results listed in Table I for the error to $g_{2 M}$ obtained from the power quadrupole moment $\hat{b}_{2 M}$ are not quite as good as this $N_{\text {pix }}^{-1 / 2}$ expectation. The origin of this discrepancy can be traced to two sources. First of all, the two-dimensional CMB signal is degraded from the three-dimensional power spectrum; a Fourier mode in the $\hat{\mathbf{z}}$ direction gives rise to some temperature fluctuation near the north pole, and not just at the equator. This is manifest in the large coefficients (e.g., the factor of $512 \pi$ ) in our analytic estimates.

However, another reason that the estimator $\hat{b}_{2 M}$ does not provide a sensitive probe of a quadrupole departure from statistical isotropy is that it is not an optimal estimator for $g_{2 M}$. This estimator sums the "signals" $D_{l l^{\prime}}^{L M}$, but it does not weight these signals properly. This can be seen by noting that for a $C_{l} \propto l^{-2}$ power spectrum, for example, the error obtained from Eq. (18) can be reduced by applying a low-pass filter: i.e., by increasing the minimum values of $l l^{\prime}$ in the sums. (A simple calculation shows 
that with the properly chosen lower- $l$ limit, $\sigma_{g_{2 M}}$ can be reduced by a factor of 30.) If the precision of the result is improved by removing data, then something is suboptimal.

\section{MINIMUM-VARIANCE ESTIMATOR}

\section{A. Estimator and its variance}

The $\hat{b}_{L M}$ estimator is a simple and intuitive quantity that can be measured to test for statistical isotropy in a modelindependent way. However, if one has a specific theory, defined by the functions $g_{L M}(k)$ or some quantities that parametrize the $g_{L M}(k)$, then there will be estimators that can be constructed to measure optimally those parameters. For example, if the $g_{L M}$ 's are all constants, then one can measure them better than the numerical results for the power multipole moments $b_{L M}$ would suggest. Below, we will derive the minimum-variance estimator for $g_{L M}$.

Before moving on, it is instructive and will be useful below to rederive the variance to $\hat{b}_{L M}^{\text {map }}$. We return to Eq. (14) and note that $b_{L M}^{\mathrm{map}}$ can be written as a sum over $D_{l l^{\prime}}^{L M \text { map }} \equiv D_{l l^{\prime}}^{L M} W_{l} W_{l^{\prime}}$. We then return to Eq. (6) to derive the minimum-variance estimator for $D_{l l^{\prime}}^{L M \text {,map }}$. Given a map $a_{l m}^{\mathrm{map}}$, each $\mathrm{mm}^{\prime}$ pair provides an estimator for $D_{l l^{\prime}}^{L M \text {,map }}$, through

$$
\hat{D}_{l l^{\prime}, m m^{\prime}}^{L M, \operatorname{map}}=\frac{a_{l m}^{\operatorname{map}} a_{l^{\prime} m^{\prime}}^{\mathrm{map}, *}-C_{l} \delta_{l l^{\prime}} \delta_{m m^{\prime}}}{\xi_{l m l^{\prime} m^{\prime}}^{L M}},
$$

with variance

$$
\left\langle\left(\hat{D}_{l l^{\prime}, m m^{\prime}}^{L M, \text { map }}\right)^{2}\right\rangle=\frac{\left(1+\delta_{l l^{\prime}} \delta_{m m^{\prime}}\right) C_{l}^{\text {map }} C_{l^{\prime}}^{\text {map }}}{\left(\xi_{l m l^{\prime} m^{\prime}}^{L M}\right)^{2}} .
$$

The estimators for different $\mathrm{mm}^{\prime}$ pairs are uncorrelated (if we use the real and imaginary parts of the $a_{l m}$ 's), so the estimators can be summed over all $\mathrm{mm}^{\prime}$ pairs, inversely weighted by the variance, to obtain a minimum-variance estimator. If $l=l^{\prime}$, we sum only over $m^{\prime} \geq m$ to avoid double-counting pairs. However, the factor $\left(1+\delta_{l l^{\prime}} \delta_{m m^{\prime}}\right)$ then weights the $m=m^{\prime}$ modes twice as much, if $l=l^{\prime}$, and thus allows us to rewrite the sum over all $m$ and $m^{\prime}$. The result for the estimator can thus be written, for both $l=l^{\prime}$ and $l \neq l^{\prime}$, as

$$
\hat{D}_{l l^{\prime}}^{L M, \text { map }}=\frac{\sum_{m m^{\prime}} a_{l m}^{\mathrm{map}} a_{l^{\prime} m^{\prime}}^{\mathrm{map} *} \xi_{l m l^{\prime} m^{\prime}}^{L M}}{G_{l l^{\prime}}^{L}} .
$$

We recognize these to be the bipolar-spherical-harmonic coefficients of Ref. [17], with a slightly different weight. The variance of this estimator is then

$$
\left\langle\left(\hat{D}_{l l^{\prime}}^{L M, \text { map }}\right)^{2}\right\rangle=\frac{\left(1+\delta_{l l^{\prime}}\right) C_{l}^{\mathrm{map}} C_{l^{\prime}}^{\text {map }}}{G_{l l^{\prime}}^{L}} .
$$

The variance, Eq. (18), with which each $b_{l m}$ can be measured simply follows by summing the variances of each term in Eq. (14).
Now, to construct the minimum-variance estimator, we simply note that the statistically independent quantities predicted by the theory are the $D_{l l^{\prime}}^{L M}$, s, the generalizations of the $C_{l}$ 's for a theory without statistical isotropy. We have constructed above estimators for these quantities, and we have their variances. For a theory with constant $g_{L M}$ 's, each $D_{l l^{\prime}}^{L M}$ provides an estimator through $\hat{g}_{L M, l l^{\prime}} \equiv$ $\hat{D}_{l l^{\prime}}^{L M} / F_{l l^{\prime}}$. We then sum these, inversely weighted by their variance to obtain the minimum-variance estimator,

$$
\hat{g}_{L M}=\frac{\sum_{l^{\prime} \geq l} F_{l l^{\prime}} W_{l} W_{l^{\prime}} \hat{D}_{l l^{\prime}}^{L M, \text { map }}\left\langle\left(\hat{D}_{l l^{\prime}}^{L M, \text { map }}\right)^{2}\right\rangle^{-1}}{\sum_{l^{\prime} \geq l}\left(F_{l l^{\prime}} W_{l} W_{l^{\prime}}\right)^{2}\left\langle\left(\hat{D}_{l l^{\prime}}^{L M, \text { map }}\right)^{2}\right\rangle^{-1}},
$$

obtained from the entire map. The variance $\sigma_{g_{L M}}^{2}$ of this estimator is then obtained by summing the inverse variances of all the estimators. Again, the sums are over $l^{\prime} \geq l$, but the factor $\left(1+\delta_{l l^{\prime}}\right)$ in Eq. (22) allows us to write the sum over all $l l^{\prime}$,

$$
\frac{1}{\sigma_{g_{L M}}^{2}}=\sum_{l l^{\prime}} G_{l l^{\prime}}^{L} \frac{\left(F_{l l^{\prime}} W_{l} W_{l^{\prime}}\right)^{2}}{2 C_{l}^{\text {map }} C_{l^{\prime}}^{\text {map }}}
$$

\section{B. Illustration: The Power Quadrupole}

To illustrate, we now evaluate this expression for $L=2$. Again, in this case, the only $l l^{\prime}$ combinations that contribute are $l^{\prime}=l$ and $l^{\prime}=l \pm 2$. We assume $l, l^{\prime} \gg 1$, approximate $F_{l, l+2} \simeq-0.5 C_{l}$, as above, and evaluate $C_{l 0 l^{\prime} 0}^{L M}$ as in Sec. IV C. We can then write,

$$
\frac{1}{\sigma_{g_{2 M}}^{2}} \simeq 0.035 \sum_{l} \frac{l C_{l}^{2}\left(W_{l}\right)^{4}}{\left(C_{l}^{\text {map }}\right)^{2}},
$$

which we can further approximate as $0.017 l_{\max }^{2}$, where $l_{\max }$ is the multipole moment at which $C_{l}^{\mathrm{n}} \simeq C_{l}\left(W_{l}\right)^{2}$. The end result is then $\sigma_{g_{2 M}} \simeq 7.6 / l_{\max }$, quite close to what we would have expected by simply counting the number $N_{\text {pix }} \simeq l_{\max }^{2}$ of usable pixels. For the WMAP and Planck temperature maps, $l_{\max }$ is roughly 650 and 2000 , respectively, implying $\sigma_{g_{2 M}} \sim 1.2 \times 10^{-2}$ and $3.8 \times 10^{-3}$, respectively, implying very significant improvements in the sensitivity over the power multipole moments.

Table I lists the exact numerical results, obtained by evaluating Eq. (24) exactly, for both TT only and EE only. Again, the Appendix generalizes Eq. (24) for the full temperature-polarization map, including the TE cross-correlation, and numerical results for this case are also included. Table I shows that by weighting the modes correctly, we get an improvement of a factor of $\sim 2$ for WMAP and Planck EE and more than an order-ofmagnitude improvement for WMAP and Planck TT; this is in accord with our arguments that the signal to noise in the TT power multipole moments was particularly poorly chosen. Although EPIC will have vastly improved instrumental sensitivity, with its modest angular resolution, it is not particularly well suited to search for departures from 
statistical isotropy. Again, the minimum-variance numbers in Table I must be increased by about $25 \%$ to account for partial-sky coverage. And again, since the preferred direction is not known a priori, the sum of the squares of the $g_{2 M}$ 's must exceed $\left(3 \sigma_{g_{2 M}}\right)^{2}$ to claim a " $3 \sigma$ " detection of a departure of statistical isotropy.

\section{CONCLUDING REMARKS}

We have considered CMB tests for the statistical isotropy of the primordial power spectrum. The power spectrum of Eq. (2) is the most general power spectrum if the assumption of statistical isotropy is dropped. In the more general case, the CMB power spectrum $C_{l}$ is generalized to a set of moments $D_{l l^{\prime}}^{L M}$, which are closely analogous to the bipolar-spherical-harmonic coefficients of Ref. [17]. The power multipole moments $b_{L M}$ provide simple and intuitive statistics that can be used to search in a modelindependent way for departures from statistical isotropy. If, however, a particular model is introduced by specifying a particular parametrization of the functions $g_{L M}(k)$, then minimum-variance statistics can be introduced to improve the precision with which these parameters can be constrained. For example, we constructed explicitly the minimum-variance estimators for the coefficients $g_{L M}$ for the case in which they are $k$ independent. We applied these results to a model in which there is a quadrupole in the primordial power spectrum, and the results are shown in Table I. We see that the best probe of a primordial quadrupole moment will come from Planck TT, for which we anticipate $\sigma_{g_{2 M}}=0.0052$. Multiplying this by 1.25 to account for a $65 \%$ sky coverage, and then by the factor of 3 required for a " $3 \sigma$ " detection, we find that the smallest quadrupole amplitude that will be detectable by Planck will be around $2.0 \%$.

To reduce clutter in the equations and to keep the main line of reasoning clear, we have derived equations in the main body of the paper for the case where either the temperature or the polarization is used, but not both. The Appendix generalizes the analysis to allow the use of the full temperature-polarization information, including the TE cross-correlation.

What about other probes? Consider, for example, the Sloan Digital Sky Survey [23]. The volume and galaxy density of the main galaxy survey allows measurement, roughly speaking, of the amplitudes of $N_{\text {modes }} \sim 10^{5}$ independent Fourier modes of the density field, in the linear regime, and these measurements are cosmic-variance limited. Measurement of the quadrupole of the power spectrum can then simply be done by comparing the amplitudes of Fourier modes in different directions. The standard error to the power multipole moments will thus be $\sigma_{g_{L M}} \sim$ $\sqrt{2 / N_{\text {modes }}} \sim 10^{-2}$, comparable in order of magnitude to what can be achieved with the CMB. Of course, a realistic search will be hampered by the irregular volume of the survey, redshift-space distortions, and anisotropies (lineof-sight versus angular) inherent to the measurement technique. But then again, there will be degradations (foregrounds, sky cuts, etc.) to the idealized CMB measurements we have considered. Of course, if $g_{L M}(k)$ varies with $k$, then the constraints provided by the CMB and galaxy surveys will be complementary, to the extent that the wave numbers $k$ probed by the CMB and galaxy surveys differ. Looking forward, there is ultimately the possibility of accessing with $21-\mathrm{cm}$ fluctuations approximately $10^{15}$ modes of the primordial density field [24], allowing values as small as $g_{L M} \sim 10^{-7}$ to be probed, but this is in the very far future.

\section{ACKNOWLEDGMENTS}

We thank D. Babich, K. Gorski, M. Wise, and C. Pahud for useful comments on an earlier draft. M. K. acknowledges the hospitality of the Aspen Center for Physics, where part of this work was completed. A. P. acknowledges the support of the NSF. This work was supported by DOE DE-FG03-92-ER40701, NASA NNG05GF69G, the Gordon and Betty Moore Foundation, and a NASA Einstein Probe mission study grant, "The Experimental Probe of Inflationary Cosmology."

\section{APPENDIX: GENERALIZATION TO A TEMPERATURE-POLARIZATION MAP}

For most experiments, the sensitivity to departures from statistical isotropy will come primarily from either the temperature or the polarization. Considering both in tandem will provide some improvement in the result, but given the temperature-polarization cross-correlation, this improvement will be weaker than what would be obtained by simply adding the two results in quadrature.

Still, to be complete, we include expressions for theory and estimators for a combined temperature-polarization map. Assuming only primordial density perturbations contribute to the temperature-polarization map, a map of the sky will now provide the E-mode polarization $E(\hat{\mathbf{n}})$, constructed in the usual fashion $[25,26]$ from the measured Stokes parameters $Q(\hat{\mathbf{n}})$ and $U(\hat{\mathbf{n}})$, in addition to the temperature $T(\hat{\mathbf{n}})$. The map can be written in terms of spherical-harmonic coefficients $a_{l m}^{\mathrm{X}}$, for $\mathrm{X}=\{\mathrm{T}, \mathrm{E}\}$, and Eq. (6) is generalized to

$$
\left\langle a_{l m}^{\mathrm{X}} a_{l^{\prime} m^{\prime}}^{\mathrm{X}^{\prime}, *}\right\rangle=\delta_{l l^{\prime}} \delta_{m m^{\prime}} C_{l}^{\mathrm{XX}}+\sum_{L M} \xi_{l m l^{\prime} m^{\prime}}^{L M} D_{l l^{\prime}}^{L M, \mathrm{XX}^{\prime}} .
$$

The $C_{l}^{\mathrm{XX}}$ 's and $D_{l l^{\prime}}^{L M, \mathrm{XX}^{\prime}}$, s are obtained as in Eqs. (7) and (8) by replacing the $\Theta_{l}(k) \Theta_{l^{\prime}}(k)$ factors in the integrands of those equations by $\Theta_{l}^{\mathrm{X}}(k) \Theta_{l}^{\mathbf{X}^{\prime}}(k)$, where these are obtained from Eq. (5) by replacing $T(\hat{\mathbf{n}})$ by $X(\hat{\mathbf{n}})$. Note that for TE and $l \neq l^{\prime}, D_{l l^{\prime}}^{L M, \mathrm{XX}^{\prime}} \neq D_{l^{\prime} l}^{L M, \mathrm{XX}^{\prime}}$. This will affect the equations below for the minimum-variance estimator. 
We now have a set of three power multipole moments $b_{L M}^{\mathrm{XX}^{\prime}}$, obtained from Eq. (11) by replacing $\left\langle T^{2}\right\rangle$ by $\left\langle X X^{\prime}\right\rangle$, which is itself obtained from Eq. (12) by using $\left\langle a_{l m}^{\mathrm{X}} a_{l^{\prime} m^{\prime}}^{\mathrm{X}^{\prime}, *}\right\rangle$ for the expectation value therein. The expression for the $b_{L M}^{\mathrm{XX}}$ is the same as Eq. (14) using $D_{l l^{\prime}}^{L M, \mathrm{XX}^{\prime}}$ there.

The power-multipole-moment estimators $\hat{b}_{L M}^{\mathrm{XX} \text { ',map }}$ are as in Eq. (15) with $\left[T^{\text {map }}(\hat{\mathbf{n}})\right]^{2}$ replaced by $\left[X^{\operatorname{map}}(\hat{\mathbf{n}}) X^{\text {map }}(\hat{\mathbf{n}})\right]$. Things get a bit trickier, though, when we calculate the variances, as the estimators for different $\mathrm{XX}^{\prime}$ will now be correlated, although still uncorrelated for different $L M$. The variance in Eq. (16) is now promoted to a $3 \times 3$ matrix $\Xi_{\mathrm{AA}^{\prime}}^{L M}$, for $\left\{\mathrm{A}, \mathrm{A}^{\prime}\right\}=\{\mathrm{TT}, \mathrm{EE}, \mathrm{TE}\}$. For $\left\{\mathrm{AA}^{\prime}\right\}=\mathrm{XX}^{\prime}=$ $\{\mathrm{TT}, \mathrm{EE}\}, \Xi_{\mathrm{AA}^{\prime}}^{L M}$ is given by Eq. (17) with $C_{l_{1}}^{\text {map }} C_{l_{2}}^{\text {map }}$ replaced by $C_{l_{1}}^{\mathrm{A} \text {,map }} C_{l_{2}}^{\mathrm{A}^{\prime} \text {,map }}$. For the diagonal TE-TE term,

$$
\Xi_{\mathrm{TE}, \mathrm{TE}}^{L M}=\sum_{l l^{\prime}} G_{l l^{\prime}}^{L}\left[C_{l}^{\mathrm{TT}, \mathrm{map}} C_{l^{\prime}}^{\mathrm{EE}, \mathrm{map}}+C_{l}^{\mathrm{TE}, \mathrm{map}} C_{l^{\prime}}^{\mathrm{TE}, \mathrm{map}}\right],
$$

and for the off-diagonal $\mathrm{XX}-\mathrm{XX}^{\prime}$ terms,

$$
\Xi_{\mathrm{XX}, \mathrm{XX}}^{L M}=2 \sum_{l l^{\prime}} G_{l l^{\prime}}^{L} C_{l}^{\mathrm{XX}, \mathrm{map}} C_{l^{\prime}}^{\mathrm{XX}, \mathrm{map}} .
$$

Equation (18) for the standard error with which a constant $g_{L M}$ can be recovered with the power multipole moments is then replaced by [25-27]

$$
\frac{1}{\sigma_{g_{L M}}^{2}}=\sum_{\mathrm{AA}^{\prime}} \frac{\partial b_{L M}^{A}}{\partial g_{L M}}\left[\left(\Xi^{L M}\right)^{-1}\right]_{\mathrm{AA}^{\prime}} \frac{\partial b_{L M}^{\mathrm{A}^{\prime} *}}{\partial g_{L M}} .
$$

This is the equation used to obtain the "total" results listed in Table I for the power multipole moment.

The minimum-variance estimator for $g_{L M}$ and its variance are similarly generalized. The estimators $\hat{D}_{l l^{\prime}}^{L M, \mathrm{~A} \text {,map }}$ are still uncorrelated for different $l l^{\prime}$ pairs and different $L M$, but they are now correlated for different $\mathrm{A}$. The main subtlety is that since $D_{l l^{\prime}}^{L M, \mathrm{TE}} \neq D_{l^{\prime} l}^{L M, \mathrm{TE}}$, we must be careful to keep track of all TE modes for $l \neq l^{\prime}$. This will require that we split the sum in the generalization of Eq. (24) into two sums: the first over $l=l^{\prime}$, and the second over $l^{\prime}>l$.
(Actually, the sum can in fact be written over all $l l^{\prime}$, but at the cost of much uglier algebraic expressions.)

For $l^{\prime}=l$, there are now three (TT, EE, and TE) estimators to replace that in Eq. (19), and for $l^{\prime}>l$, there are now four (TT, EE, TE, and ET) estimators to replace that in Eq. (19). For all $l l^{\prime}$, the estimators are as in Eq. (19), replacing each $a_{l m}^{\text {map }}$ and $C_{l}^{\text {map }}$ by the appropriate $a_{l m}^{\mathrm{X} \text {,map }}$ and $C_{l}^{\mathrm{XX}}$,map, respectively. The estimator for each $l l^{\prime}$, obtained after summing over all $\mathrm{mm}^{\prime}$, is the same as in Eq. (21). For $l=l^{\prime}$, the variances $\left\langle\left(\hat{D}_{l l^{\prime}}^{L M, \mathrm{~A}, \mathrm{map}}\right)^{2}\right\rangle$ are now promoted to a $3 \times 3$ covariance matrix; and for $l^{\prime}>l$, they are promoted to a $4 \times 4$ covariance matrix. In both cases, the covariance matrix can be written as

$$
\mathcal{C}_{\mathrm{AA}^{\prime}}^{l l^{\prime}} \equiv \frac{G_{l l^{\prime}}^{L}}{\left(1+\delta_{l l^{\prime}}\right)}\left\langle\hat{D}_{l l^{\prime}}^{L M, \mathrm{~A}, \mathrm{map}} \hat{D}_{l l^{\prime}}^{L M, \mathrm{~A}^{\prime}, \mathrm{map}}\right\rangle .
$$

For any $l l^{\prime}$ pair, the diagonal entries, for $\mathrm{A}=\{\mathrm{TT}, \mathrm{EE}\}$, are $\mathcal{C}_{\mathrm{AA}}^{l l^{\prime}}=C_{l}^{\mathrm{A} \text {,map }} C_{l^{\prime}}^{\mathrm{A} \text {,map }}$, and the TT-EE off-diagonal entry is $\mathcal{C}_{\mathrm{TT}, \mathrm{EE}}^{l l^{\prime}}=C_{l}^{\mathrm{TE}, \mathrm{map}} C_{l^{\prime}}^{\mathrm{TE} \text {,map }}$. For $l=l^{\prime}$, the diagonal TE-TE entry is

$$
\mathcal{C}_{\mathrm{TE}, \mathrm{TE}}^{l l^{\prime}}=\left[C_{l}^{\mathrm{TT}, \mathrm{map}} C_{l}^{\mathrm{EE}, \text { map }}+\left(C_{l}^{\mathrm{TE}, \mathrm{map}}\right)^{2}\right] / 2 .
$$

For $l^{\prime}>l$, we have $\mathcal{C}_{\mathrm{TE}, \mathrm{TE}}^{l l^{\prime}}=C_{l}^{\mathrm{TT}} C_{l^{\prime}}^{\mathrm{EE}}, \mathcal{C}_{\mathrm{ET}, \mathrm{ET}}^{l l^{\prime}}=C_{l^{\prime}}^{\mathrm{TT}} C_{l}^{\mathrm{EE}}$, and $\mathcal{C}_{\mathrm{TE}, \mathrm{ET}}^{l l^{\prime}}=C_{l}^{\mathrm{TE}} C_{l^{\prime}}^{\mathrm{TE}}$. For any $l l^{\prime}$, we have $\mathcal{C}_{\mathrm{TT}, \mathrm{TE}}^{l l^{\prime}}=$ $C_{l}^{\mathrm{TT}} C_{l^{\prime}}^{\mathrm{TE}}$ and $\mathcal{C}_{\mathrm{EE}, \mathrm{TE}}^{l^{\prime}}=C_{l^{\prime}}^{\mathrm{EE}} C_{l}^{\mathrm{TE}}$. For $l^{\prime}>l$, we also have $\mathcal{C}_{\mathrm{TT}, \mathrm{ET}}^{l^{\prime}}=C_{l^{\prime}}^{\mathrm{TT}} C_{l}^{\mathrm{TE}}$ and $\mathcal{C}_{\mathrm{EE}, \mathrm{ET}}^{l l^{\prime}}=C_{l}^{\mathrm{EE}} C_{l^{\prime}}^{\mathrm{TE}}$.

The generalization of Eq. (24) is then

$$
\begin{aligned}
\frac{1}{\sigma_{g_{L M}}^{2}}= & \frac{1}{2} \sum_{l} G_{l l}^{L} \sum_{A A^{\prime}} C_{l}^{\mathrm{A}} C_{l}^{\mathrm{A}^{\prime}}\left(W_{l}\right)^{4}\left[\left(\mathcal{C}^{l l}\right)^{-1}\right]_{\mathrm{AA}^{\prime}} \\
& +\sum_{l^{\prime}>l} G_{l l^{\prime}}^{L} \sum_{\mathrm{AA}^{\prime}} F_{l l^{\prime}}^{\mathrm{A}} F_{l l^{\prime}}^{\mathrm{A}^{\prime}}\left(W_{l} W_{l^{\prime}}\right)^{2}\left[\left(\mathcal{C}^{l l^{\prime}}\right)^{-1}\right]_{\mathrm{AA}^{\prime}},
\end{aligned}
$$

where the matrix inversion is in the $3 \times 3 \mathrm{AA}^{\prime}$ space in the first sum and in the $4 \times 4 \mathrm{AA}^{\prime}$ space in the second sum. We use Eq. (A7) to evaluate the standard errors for the total minimum-variance estimators listed in Table I.
[1] A. de Oliveira-Costa, M. Tegmark, M. Zaldarriaga, and A. Hamilton, Phys. Rev. D 69, 063516 (2004); C. J. Copi, D. Huterer, and G. D. Starkman, Phys. Rev. D 70, 043515 (2004); D. J. Schwarz, G. D. Starkman, D. Huterer, and C. J. Copi, Phys. Rev. Lett. 93, 221301 (2004); S. Prunet, J. P. Uzan, F. Bernardeau, and T. Brunier, Phys. Rev. D 71, 083508 (2005); H. K. Eriksen, A. J. Banday, K. M. Gorski, and P. B. Lilje, Astrophys. J. 622, 58 (2005); K. Land and J. Magueijo, Phys. Rev. Lett. 95, 071301 (2005); T. R. Jaffe, A. J. Banday, H. K. Eriksen, K. M. Gorski, and F. K.
Hansen, Astrophys. J. 629, L1 (2005); C. J. Copi, D. Huterer, D. J. Schwarz, and G. D. Starkman, Mon. Not. R. Astron. Soc. 367, 79 (2006); K. Land and J. Magueijo, Mon. Not. R. Astron. Soc. 367, 1714 (2006); A. Bernui, T. Villela, C. A. Wuensche, R. Leonardi, and I. Ferreira, Astron. Astrophys. 454, 409 (2006); L. R. Abramo, A. Bernui, I. S. Ferreira, T. Villela, and C.A. Wuensche, Phys. Rev. D 74, 063506 (2006); J. Magueijo and R. D. Sorkin, Mon. Not. R. Astron. Soc. 377, L39 (2007); C. G. Park, C. Park, and J.R. I. Gott, Astrophys. J. 660, 959 
(2007); D. Huterer, New Astron. Rev. 50, 868 (2006); P. Vielva, Y. Wiaux, E. Martinez-Gonzalez, and P. Vandergheynst, New Astron. Rev. 50, 880 (2006); K. Land and J. Magueijo, Mon. Not. R. Astron. Soc. 378, 153 (2007); C. Gordon, W. Hu, D. Huterer, and T. Crawford, Phys. Rev. D 72, 103002 (2005); J.G. Cresswell, A. R. Liddle, P. Mukherjee, and A. Riazuelo, Phys. Rev. D 73, 041302 (2006); S. H.S. Alexander, arXiv:hep-th/0601034.

[2] H. K. Eriksen, F. K. Hansen, A. J. Banday, K. M. Gorski, and P. B. Lilje, Astrophys. J. 605, 14 (2004); 609, 1198(E) (2004).

[3] F. K. Hansen, A. J. Banday, and K. M. Gorski, arXiv:astro$\mathrm{ph} / 0404206$.

[4] H. K. Eriksen, A. J. Banday, K. M. Gorski, F. K. Hansen, and P. B. Lilje, Astrophys. J. 660, L81 (2007).

[5] G. V. Chibisov and Y. V. Shtanov, Sov. Phys. JETP 69, 17 (1989).

[6] G. V. Chibisov and Y. V. Shtanov, Int. J. Mod. Phys. A 5, 2625 (1990).

[7] A. Berera, R. V. Buniy, and T. W. Kephart, J. Cosmol. Astropart. Phys. 10 (2004), 016.

[8] E. P. Donoghue and J.F. Donoghue, Phys. Rev. D 71, 043002 (2005).

[9] R. V. Buniy, A. Berera, and T. W. Kephart, Phys. Rev. D 73, 063529 (2006).

[10] L. Ackerman, S. M. Carroll, and M. B. Wise, Phys. Rev. D 75, 083502 (2007).

[11] A.E. Gumrukcuoglu, C. R. Contaldi, and M. Peloso, arXiv:0707.4179.

[12] C. Armendariz-Picon, arXiv:0705.1167.

[13] J.F. Donoghue, K. Dutta, and A. Ross, arXiv:astro-ph/
0703455.

[14] T. S. Pereira, C. Pitrou, and J.-P. Uzan, J. Cosmol. Astropart. Phys. 09 (2007) 006.

[15] R. A. Battye and A. Moss, Phys. Rev. D 74, 041301 (2006).

[16] T. Koivisto and D.F. Mota, arXiv:0707.0279.

[17] A. Hajian and T. Souradeep, Astrophys. J. 597, L5 (2003); S. Basak, A. Hajian, and T. Souradeep, Phys. Rev. D 74, 021301 (2006); A. Hajian and T. Souradeep, arXiv:astro$\mathrm{ph} / 0501001$.

[18] C. Armendariz-Picon, J. Cosmol. Astropart. Phys. 03 (2006) 002.

[19] U. Seljak and M. Zaldarriaga, Astrophys. J. 469, 437 (1996); cfa-www.harvard.edu/ mzaldarr/CMBFAST/ cmbfast.html.

[20] map.gsfc.nasa.gov.

[21] Planck Collaboration, arXiv:astro-ph/0604069.

[22] J. Bock et al. (unpublished).

[23] www.sdss.org.

[24] A. Loeb and M. Zaldarriaga, Phys. Rev. Lett. 92, 211301 (2004).

[25] M. Kamionkowski, A. Kosowsky, and A. Stebbins, Phys. Rev. D 55, 7368 (1997); M. Kamionkowski, A. Kosowsky, and A. Stebbins, Phys. Rev. Lett. 78, 2058 (1997).

[26] M. Zaldarriaga and U. Seljak, Phys. Rev. D 55, 1830 (1997); U. Seljak and M. Zaldarriaga, Phys. Rev. Lett. 78, 2054 (1997).

[27] G. Jungman, M. Kamionkowski, A. Kosowsky, and D. N. Spergel, Phys. Rev. D 54, 1332 (1996); G. Jungman, M. Kamionkowski, A. Kosowsky, and D. N. Spergel, Phys. Rev. Lett. 76, 1007 (1996). 